\title{
Idiopathic nodular glomerulosclerosis- a rare case
}

\author{
Jazmin I. Yepez Kuri MD, Carlos L. Torres Roman MD, \\ Anand Reddy MD, Yasir Ahmed MD
}

\begin{abstract}
Nodular glomerulosclerosis is considered a signature lesion of diabetes nephropathy, but when it occurs in the absence of diabetes, it is called Idiopathic Nodular Glomerulosclerosis (ING). Idiopathic nodular glomerulosclerosis is a very rare disease and has been associated with obesity, hypertension, and smoking. We report a case of ING presenting as end-stage renal failure requiring hemodialysis.
\end{abstract}

Key words: idiopathic, nodular glomerulosclerosis, nephropathy

\section{INTRODUCTION}

Nodular glomerulosclerosis is considered a pathognomonic sign of diabetic nephropathy. ${ }^{1}$ Millions of patients worldwide are affected with this nephropathy. ${ }^{1,2}$ Idiopathic nodular glomerulosclerosis (ING) is very rare, and fewer than 50 cases have been reported in the English literature. ${ }^{3}$ It is considered a distinct nephropathy with light microscopy and ultrastructural changes that mimic diabetic nodular glomerulosclerosis but without impaired glucose metabolism or other specific disease characteristics. ${ }^{4,5}$ Here we report a case of ING in an obese patient presenting with endstage renal disease.

Corresponding author: Yasir Ahmed MD Contact Information: Yasir.ahmed@ttuhsc.edu DOI: $10.12746 /$ swrccc2016.0416.223

\section{CASE}

A 34-year-old Hispanic woman with an increased body mass index (BMI, $33.7 \mathrm{Kg} / \mathrm{m}^{2}$ ), hypothyroidism, and kidney disease presented to the emergency room with headache, generalized weakness, and new onset hypertension. She had no recent history of infection or use of OTC nonsteroidal anti-inflammatory drugs. During admission, she looked sick; her blood pressure was 150/80 mmHg. Funduscopic examination revealed vitreous hemorrhages and cataracts in both eyes.

The initial laboratory studies included serum creatinine $12.07 \mathrm{mg} / \mathrm{dL}$, blood urea nitrogen $99 \mathrm{mg} /$ $\mathrm{dL}$, estimated glomerular filtration rate $4 \mathrm{~mL} / \mathrm{min} / 1.73$ $\mathrm{m}^{2}$, urine protein $14.1 \mathrm{~g} / 24 \mathrm{hr}$, serum albumin 2.3 $\mathrm{g} / \mathrm{dL}$, total proteins $5.4 \mathrm{~g} / \mathrm{dL}$, serum potassium 5.2 $\mathrm{mmol} / \mathrm{L}$, hemoglobin $8.5 \mathrm{gm} / \mathrm{dL}$, hematocrit $24.8 \%$, and $\mathrm{HbA} 1 \mathrm{c} 5.1 \%$. Antiglomerular base membrane antibodies, C-ANCA, P-ANCA, atypical pANCA, complement $\mathrm{C} 3$ and $\mathrm{C} 4$ levels, hepatitis B surface antigen, and $\mathrm{C}$ antibody assays were either within normal limits or negative. 
Renal biopsy revealed advanced nodular glomerulosclerosis with severe tubular atrophy with interstitial fibrosis, and severe arterio- and arteriolosclerosis. By light microscopy the specimen had 17 glomeruli; 15 were globally sclerotic. Electron microscopy confirmed the sclerotic glomeruli were large with mesangial nodules, the glomerular basement membrane was diffusely thickened and wrinkled, and the capillaries were narrowed and obliterated due to the increased mesangial matrix. The fluoresceinated antibody stains showed linear capillary wall staining for $\operatorname{lgG}(1+)$ and albumin (1+). A Congo red stain was negative for amyloidosis. Given the lack of history of diabetes, the morphologic features were consistent with idiopathic nodular glomerulosclerosis (Figure $1 \mathrm{~A}, 1 \mathrm{~B}$ and $1 \mathrm{C}$ ). She was started on intermittent hemodialysis and has been maintained on thrice weekly dialysis.

\section{Discussion}

Idiopathic nodular glomerulosclerosis was first described by Alpers and Biava as a rare clinicalpathologic entity in five patients without diabetes mellitus. ${ }^{6}$ In 1999, Herzenberg et al used the term "Idiopathic nodular glomerulosclerosis" to describe the histopathologic findings of renal biopsy that mimic diabetic nodular glomerulosclerosis. Since then numerous associations have been linked with this rare entity. ${ }^{7}$ Exogenous factors, such as smoking, endogenous factors, such as hypertension, obesity, or hypercholesterolemia, biochemical events caused by hormonal responses or cytokines, and demographic factors, such as older age, white race/ethnicity, and male gender, have been linked to IGN.

Although we have little information about this patient's past history, the most interesting feature is her young age (34 years old). Most patients reported in the literature are white males with a mean age above 55 years and chronic hypertension and smoking. ${ }^{3,8-10}$ Our patient had none of these risk factors but was obese which is a common characteristic and risk factor for ING. ${ }^{8,9} \mathrm{Li}$ et al reported a series of 15 patients and found $87 \%$ of them were overweight

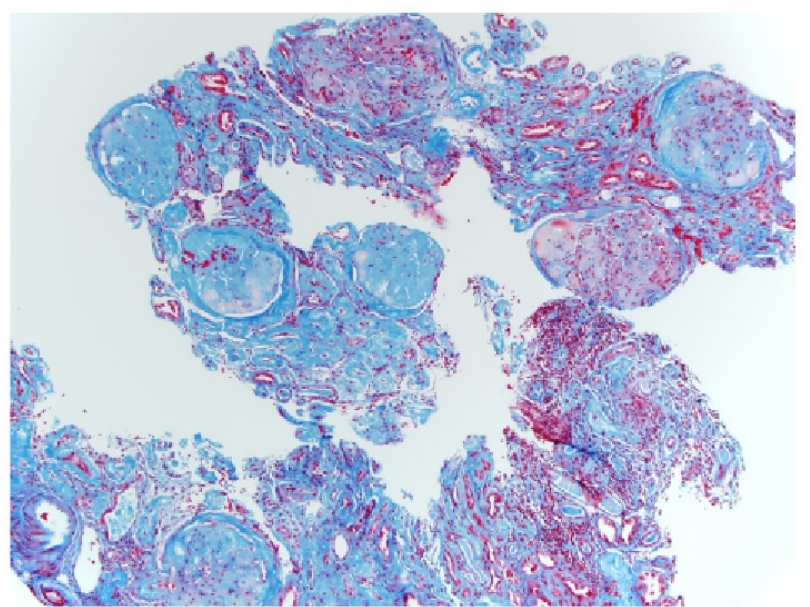

$1 \mathrm{~A}$

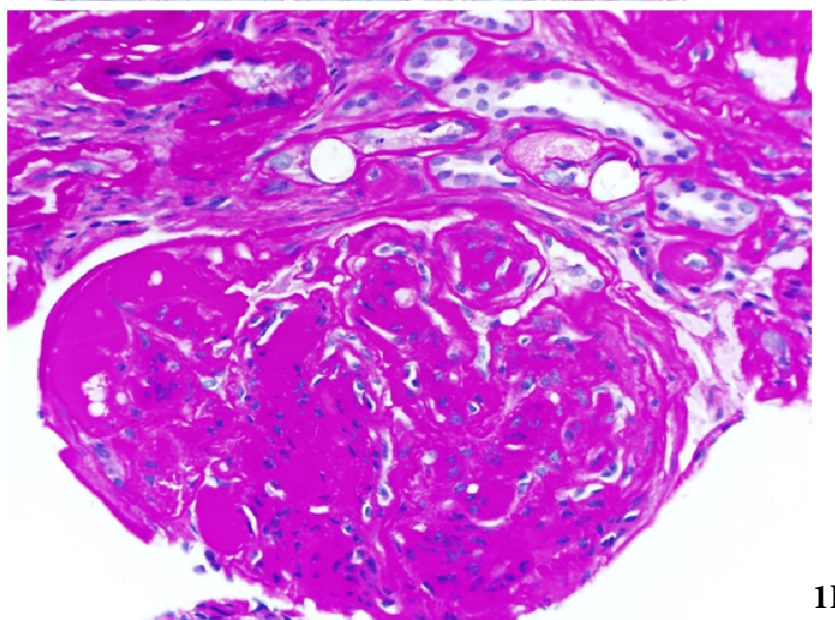

1B

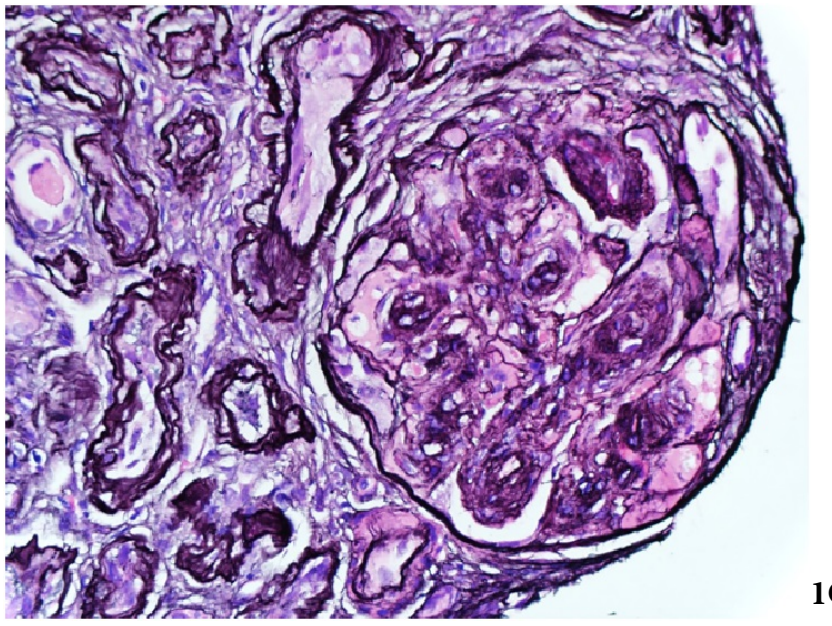

Figure 1A-C: Three small fragments of renal cortex with up to 17 glomeruli, 15 glomeruli are sclerotic. Many of the globally sclerotic glomeruli remain large with mesangial nodules still apparent. There is marked tubular atrophy and interstitial fibrosis (1A Trichrome x 100; 1B PAS x 400; 1C Jones x 400). 
or obese. ${ }^{8}$ Jun Wu et al reported a series of 20 Chinese patients with $95 \%$ being overweight or obese. ${ }^{10}$ In 2001, Kambhan et al described obesity-related glomerulopathy, a syndrome that is increasing in prevalence due to the obesity epidemic worldwide. ${ }^{15}$ Obesity-related glomerulopathy presents the same pathologic features as ING, including glomerulosclerosis, mesangial matrix expansion, and thickening of the glomerular basement.

The $\mathrm{Hb}$ A1c was $5.1 \%$ which rules out diabetes mellitus (DM) in our patient, but an oral glucose tolerance test (OGTT) was not done. Nevertheless, one possible hypothesis is that some patients have lower thresholds for glucose induced kidney damage without overt DM. ${ }^{11,16,17}$ The ophthalmological examination in our patient might suggest a hyperglycemic state like latent diabetes mellitus or a non-persistent hyperglycemic state. Navaneethan et al compiled information of 42 ING patients. Among these patients, 35 underwent OGTT, and 12 patients had glucose intolerance. One third of these patients (including those with glucose tolerance and without glucose tolerance) had diabetic retinopathy changes. ${ }^{18}$ Most ING patients develop end-stage renal disease and require dialysis. ${ }^{7,810}$ The pathologic changes in our case are among the most severe in the reported cases, and more than $80 \%$ of the glomeruli studied were globally sclerotic with marked tubular atrophy and interstitial fibrosis.

In summary, our patient lacks some of the most common risk factors for ING, such as older age, male gender, chronic hypertension, and a smoking history, but she was obese and had features of diabetic eye disease. Idiopathic nodular glomerulosclerosis is a rare disease; more studies are needed to understand its pathogenesis and possible approaches to prevention.
Author Affiliation: Jazmin I. Yepez Kuri was a fellow in Geriatrics at Texas Tech University Health Sciences Center in Odessa, TX. Carlos L. Torres Roman was a medical student in Odessa, TX. Anand Reddy and Yasir Ahmed are faculty members in the Department of Internal Medicine at TTUHSC in Odessa, TX.

Received: 05/26/2016

Accepted: 09/23/2016

Reviewers: Sorot Phisitkul MD

Published electronically: 10/15/2016

Conflict of Interest Disclosures: none

\section{REFERENCES}

1. Vinod PB. Pathophysiology of diabetic nephropathy. Clinical Queries: Nephrology 2012; 0102: 121-126.

2. Rossing P. Diabetic nephropathy: worldwide epidemic and effects of current treatment on natural history. Curr Diab Rep 2006, 6: 479-483.

3. Nasr SH, D'Agati VD. Nodular glomerulosclerosis in the nondiabetic smoker. J Am Soc Nephrol 2007; 18: 2032-2036.

4. Markowitz GS, Lin J, Valeri AM, Avila C, Nasr SH, D'Agati VD. Idiopathic nodular glomerulosclerosis is a distinct clinicopathologic entity linked to hypertension and smoking. Hum Pathol 2002; 33: 826-35.

5. Müller-Höcker J, Weiss M, Thoenes GH, Grund A, Nerlich A. A case of idiopathic nodular glomerulosclerosis mimicking diabetic glomerulosclerosis (Kimmelstiel-Wilson type). Pathol Res Pract 2002; 198: 375-379.

6. Alpers CE, Biava CG. Idiopathic lobular glomerulonephritis (nodular mesangial sclerosis): a distinct diagnostic entity. Clin Nephrol 1989; 32 (2): 68-74.

7. Holden JK, Singh S, Magil AB. Idiopathic nodular glomerulosclerosis. Am J Kidney Dis 1999; 34: 560-564.

8. Li W, Verani RR. Idiopathic nodular glomerulosclerosis: a clinopathologic study of 15 cases. Human Pathology 2008; 39: 1771-76.

9. Markowitz GS, Lin J, Valeri AM, Avila C, Nasr SH, D'Agati VD. Idiopathic nodular glomerulosclerosis is a distinct clinopathologic entity linked to hypertension and smoking. Human pathology 2002; 33: 826-835.

10. Wu J, Yu S, Tejwani V, Mao M, Muriithi AK, Ye C, Zhao X, $\mathrm{Gu} \mathrm{H}$, Mei C, Qian Q. Idiopathic nodular glomerulosclerosis in Chinese patients: a clinicopathologic study of 20 cases. Clin Exp 
Nephrol 2014; 18: 865-75.

11. Souraty P, Nast CC, Mehrotra R, Barba L, Martina J, Adler SG. Nodular glomerulosclerosis in a patient with metabolic syndrome without diabetes. Nat Clin Pract Nephrol 2008; 4: 639642.

12. Kuppachi S, Idris N, Chander PN, Yoo J. Idiopathic nodular glomerulosclerosis in a non-diabetic hypertensive smoker-case report and review literature. Nephrol Dial Transplant 2006; 21: 3571-3575.

13. Alsaad KO, Herzenberg AM. Distinguishing diabetic nephropathy from other causes of glomerulosclerosis: an update. $J$ Clin Pathol 2007; 60: 18-26.

14. Liang KV, Greene EL, Oei LS, Lewin M, Lager D, Sethi S. Nodular glomerulosclerosis: renal lesions in chronic smokers mimic chronic thrombotic microangiopathy and hypertensive lesions. Am J Kidney Dis 2007; 49: 552-559.

15. Kambham N, Markowitz GS, Valeri AM, Lin J, D'Agati VD. Obesity-related glomerulopathy: an emergent epidemic. Kidney Int 2001; 59: 1498-1509.

16. Uchida $T$, Oda $T$, Watanabe A, Higashi K, Katsurada $Y$, Shimazaki H, Tamai S, Kumagai H. Idiopathic nodular glomerulosclerosis in a never smoking, normotensive, non-obese, normal-glucose-tolerant middle-aged woman. Clin Kidney J 2012; 5: 445-448.

17. Altiparmak MR, Pamuk ON, Pamuk GE, Apaydin S, Ozbay G. Diffuse diabetic glomerulosclerosis in a patient with impaired glucose tolerance: report on a patient who later develops diabetes mellitus. Neth J Med 2002 Jul; 60(6):260-2.

18. Navaneethan SD, Singh S, Choudhry W. Nodular glomerulosclerosis in a non-diabetic patient: Case report and review of literature. J Nephrol 2005; 18: 613-615. 